CPT-93/P.2920

\title{
Chiral Defect Fermions and the Layered Phase
}

\author{
C.P. Korthals-Altes, S. Nicolis and J. Prades ${ }^{a)}$ \\ Centre de Physique Théorique, C.N.R.S. - Luminy, Case 907 \\ F-13288 Marseille Cedex 9, France
}

\begin{abstract}
Chiral defect fermions on the lattice in $4+1$ dimensions are analyzed using mean field theory. The fermion propagator has a localized chiral mode in weak coupling but loses it when the coupling in the unphysical fifth direction becomes too large. A layered phase à la Fu-Nielsen appears where the theory is vector-like in every layer.
\end{abstract}

June 1993

CPT-93/P.2920

a) Address after October 1st: NORDITA, Blegdamsvej 17

DK-2100 Copenhagen $\varnothing$, Denmark 


\section{Introduction}

A gauge invariant theory with chiral fermions is notoriously hard to construct. There is a famous obstruction to having free chiral fermions |1, 2] with a local, hermitian and translation-invariant action on the lattice and many attempts to circumvent it have been made (for a review cf. [3]).

Recently appeared a proposal [四], in which the theory is, a priori, a vector theory in $4+1$ dimensions. The mass of the fermions depends on the extra coordinate and and jumps from $-m$ to $+m$. For sufficiently small gauge coupling and smooth gauge field configurations, the presence of a chiral zero mode localized at the jump was demonstrated [5]. Its chiral partner is pushed to the boundaries in the $x_{5} \equiv \xi$ direction. Precisely how depends on the boundary conditions.

The idea now is to decouple all fermionic modes from the system except for the chiral zero mode at the defect and be left there with a chiral gauge theory. We will discuss this in the second Section.

In the next Section we will explore the phase diagram defined by the physical gauge coupling $\beta$ and the "unphysical" gauge coupling $\beta^{\prime}$. The latter couples the potential in the fifth direction. Our technique will be mean field theory. For the pure gauge case this was done by $\mathrm{Fu}$ and Nielsen [6], who discovered a layered phase for $\beta^{\prime}$ sufficiently small and $\beta$ sufficiently large. In this phase subsequent four-dimensional hyperplanes do not interact and a mean field analysis shows that fermions are propagating inside every hyperplane. The theory has become local in this phase, hence the Nielsen-Ninomiya theorem strikes again: we are left in each plane with a vector theory, thereby vetoing a proposal of ref. [4] that a viable chiral theory results at $\beta^{\prime}=0$.

In the last Section we discuss how to proceed further in the weak coupling phase using mean field analysis.

\section{Weak coupling and chiral zero modes}

In this Section we briefly review work done in refs. [4, 7, 8]. The system we study is a $U(1)$ theory coupled vectorially to Wilson fermions 


$$
\begin{gathered}
S(U, \Psi, \bar{\Psi})=\sum_{x}\left\{\beta \sum_{\mu, \nu}^{4}\left(\mathbf{1}-\operatorname{Re} U_{\mu \nu}(x)\right)+\beta^{\prime} \sum_{\mu=1}^{4}\left(\mathbf{1}-\operatorname{Re} U_{\mu 5}(x)\right)\right. \\
+\sum_{\mu=1}^{5} \bar{\Psi}(x)\left[\frac{\gamma_{\mu}}{2}\left(U_{\mu}(x) \Psi(x+\hat{\mu})-U_{\mu}^{\dagger}(x-\hat{\mu}) \Psi(x-\hat{\mu})\right)\right. \\
\left.+\frac{r}{2}\left(U_{\mu}(x) \Psi(x+\hat{\mu})-2 \Psi(x) U_{\mu}^{\dagger}(x-\hat{\mu}) \Psi(x-\hat{\mu})\right)\right] \\
+M(\xi) \bar{\Psi}(x) \Psi(x)\} .
\end{gathered}
$$

and we have that

$$
M(\xi) \equiv m \operatorname{sign}\left(\xi+\frac{1}{2}\right) .
$$

Our notation is standard. Note that the dimensionless Dirac mass is in lattice units. Let us look at the Dirac equation following from (1), setting for the moment $U_{\mu}(x)=1$. It reads

$$
\begin{gathered}
\sum_{\mu=1}^{5}\left\{\frac{\gamma_{\mu}}{2}(\Psi(x+\hat{\mu})-\Psi(x-\hat{\mu}))+\frac{r}{2}(\Psi(x+\hat{\mu})-2 \Psi(x)+\Psi(x-\hat{\mu}))\right\} \\
+M(\xi) \Psi(x)=\lambda \Psi(x) .
\end{gathered}
$$

We search for a normalizable zero mode $(\lambda=0)$ [9]. Because of translation invariance in the first four directions, we can analyze eq. (3) in terms of dimensionless lattice momenta $-\pi \leq p_{\mu}<\pi, \mu=1,2,3,4$. The result, after some trivial reshuffling, is

$$
\begin{array}{r}
\sum_{\mu=1}^{4} \mathrm{i} \gamma_{\mu} \sin p_{\mu} \Psi(p, \xi)+\frac{\gamma_{5}+r}{2} \Psi(p, \xi+1)+\frac{-\gamma_{5}+r}{2} \Psi(p, \xi-1)= \\
\left(r+r \sum_{\mu=1}^{4}\left(1-\cos p_{\mu}\right)-M(\xi)\right) \Psi(p, \xi) .
\end{array}
$$

For $r=1$, eq. (四) separates into positive and negative helicities and, for any doubler momentum $p^{D}$ (with $p_{\mu}^{D}=0$ or $\pi$ ), we get

$$
\Psi_{R, L}(p, \xi \pm 1)=\left(1+\sum_{\mu=1}^{4}\left(1-\cos p_{\mu}^{D}\right)-M(\xi)\right) \Psi_{R, L}(p, \xi)
$$

where $\Psi_{R, L} \equiv \frac{1 \pm \gamma_{5}}{2} \Psi$. Let us analyze the right-handed case. For $\xi>0$ the factor multiplying $\Psi_{R}(x)$ on the right-hand side of eq. (5) should be smaller than 1 in absolute value 


$$
\sum_{\mu=1}^{4}\left(1-\cos p_{\mu}^{D}\right)<m<2+\sum_{\mu=1}^{4}\left(1-\cos p_{\mu}^{D}\right) .
$$

Equation (6) tells us that the "doubler" $p^{D}=(0,0,0,0)$ has a normalizable righthanded zero mode only for $0<m<2$. For $2<m<4$ there are four normalizable left-handed zero modes, corresponding to the four doublers $p^{D}=(\pi, 0,0,0)$, etc.

In conclusion, eq. (6) says that for any fixed values of $m$ there is a definite number of normalizable zero modes with well-defined chirality.

The zero mode becomes visible as a pole in the propagator of the fermion, as shown by authors of ref. [10]. Taking as example $p^{D}=(0,0,0,0)$ and a physical momentum $\bar{p}$ so that $p_{\mu} \equiv a \bar{p}_{\mu} \rightarrow 0$, one finds a right-handed pole localized around $\xi=0$ in the fermion propagator $G(p)_{\xi, \xi^{\prime}}$. The above analysis is valid for $U_{\mu}=\mathbf{1}$, i.e. $\beta=\beta^{\prime}=\infty$. In the next Section we will see that the propagator behaves in a radically different fashion when $\beta^{\prime} \rightarrow 0$.

\section{Mean field approximation}

In this Section we analyze the physics of eq. (1) by mean field techniques [11]. To this end we first fix the gauge $U_{4}(x) \equiv \mathbf{1}$ everywhere. Then we write the free energy as a path integral

$$
e^{-F}=\int \mathcal{D} \bar{\Psi} \mathcal{D} \Psi \mathcal{D} U \exp -S(U, \Psi, \bar{\Psi})
$$

and gauge fixing is tacitly understood.

The next step is to introduce complex variables $v_{\mu}(x)$ by

$$
\int \mathcal{D} v \prod_{x, \mu \neq 4} \delta\left(v_{\mu}(x)-U_{\mu}(x)\right)=1
$$

and the corresponding "driving field" $\alpha_{\mu}(x)$ through the integral representation of the $\delta$-function

$$
1=\int \mathcal{D} v \int_{-\mathrm{i} \infty}^{\mathrm{i} \infty} \mathcal{D} \alpha \exp \left(-\sum_{x, \mu \neq 4} \alpha_{\mu}(x)\left(v_{\mu}(x)-U_{\mu}(x)\right)\right) .
$$

Inserting eq. (9) into eq.(7) decouples the integrals over the $U_{\mu}$. Carrying out that integration one finds

$$
e^{-F}=\int \mathcal{D} \bar{\Psi} \mathcal{D} \Psi \mathcal{D} v \mathcal{D} \alpha \exp -S_{e f f}(v, \alpha, \Psi, \bar{\Psi})
$$

with 


$$
\begin{aligned}
S_{e f f}(v, \alpha, \Psi, \bar{\Psi})= & \sum_{x}\left[\beta \sum_{\mu, \nu}^{4}\left(\mathbf{1}-\operatorname{Re} v_{\mu \nu}(x)\right)+\beta^{\prime} \sum_{\mu=1}^{4}\left(\mathbf{1}-\operatorname{Re} v_{\mu 5}(x)\right)\right. \\
& +\sum_{\mu=1}^{5} \bar{\Psi}(x)\left\{\frac{\gamma_{\mu}+r}{2} v_{\mu}(x) \Psi(x+\hat{\mu})\right. \\
+ & \left.\left.\frac{-\gamma_{\mu}+r}{2} v_{\mu}^{\dagger}(x-\hat{\mu}) \Psi(x-\hat{\mu})+(-5 r+M(\xi)) \Psi(x)\right\}\right] .
\end{aligned}
$$

We will be interested in varying $\beta$ and $\beta^{\prime}$ independently and search for a saddle point in order to evaluate eq. (10). The mean field approximation consists in setting

$$
\begin{gathered}
\left\langle U_{\mu}(x)\right\rangle=v(\xi) \quad \text { for } \quad \mu=1,2,3 \\
\text { and } \\
\left\langle U_{5}(x)\right\rangle=v^{\prime}(\xi) ;
\end{gathered}
$$

due to four-dimensional hypercubic invariance. Moreover, we will assume the saddle point is real. Under these conditions the saddle point equations read

$$
\begin{gathered}
4 \beta v^{3}(\xi)+2 \beta v(\xi)+\beta^{\prime}\left(v(\xi+1) v^{\prime 2}(\xi)+v(\xi-1) v^{\prime 2}(\xi-1)\right)+j_{\mu}(\xi)=\alpha(\xi) \\
\text { and } \\
6 \beta^{\prime} v^{\prime}(\xi) v(\xi) v(\xi+1)+2 \beta^{\prime} v^{\prime}(\xi)+j_{5}(\xi)=\alpha^{\prime}(\xi) .
\end{gathered}
$$

The relation between the mean field and the "driving field" is:

$$
\begin{aligned}
& \frac{I_{1}(\alpha(\xi))}{I_{0}(\alpha(\xi))}=v(\xi) \\
& \quad \text { and } \\
& \frac{I_{1}\left(\alpha^{\prime}(\xi)\right)}{I_{0}\left(\alpha^{\prime}(\xi)\right)}=v^{\prime}(\xi)
\end{aligned}
$$

with

$$
I_{k}(\alpha)=\int_{-\pi}^{\pi} \frac{d \phi}{2 \pi}(\cos \phi)^{k} e^{\alpha \cos \phi} .
$$

The fermionic current terms $j_{\mu}(\xi) \mu=1, \ldots, 5$ are short-hand for

$$
j_{\mu}(x) \equiv\left\langle\bar{\Psi}(x) \frac{\gamma_{\mu}+r}{2} \Psi(x+\hat{\mu})\right\rangle+\left\langle\bar{\Psi}(x+\hat{\mu}) \frac{-\gamma_{\mu}+r}{2} \Psi(x)\right\rangle
$$


and they are evaluated using the self-consistent fermion propagator

$$
G\left(x, x^{\prime}\right) \equiv\left\langle\bar{\Psi}(x) \Psi\left(x^{\prime}\right)\right\rangle
$$

with

$$
\begin{aligned}
& G^{-1}\left(x, x^{\prime}\right)=\sum_{\mu=1}^{4}\left\{v(\xi) \frac{\gamma_{\mu}+r}{2} \delta_{\xi, \xi^{\prime}} \delta_{\bar{x}+\hat{\mu}, \bar{x}^{\prime}}^{(4)}+v(\xi) \frac{-\gamma_{\mu}+r}{2} \delta_{\xi, \xi^{\prime}} \delta_{\bar{x}-\hat{\mu}, \bar{x}^{\prime}}^{(4)}\right\} \\
&+ v^{\prime}(\xi) \frac{\gamma_{5}+r}{2} \delta_{\xi+1, \xi^{\prime}} \delta_{\bar{x}, \bar{x}^{\prime}}^{(4)}+v^{\prime}(\xi-1) \frac{-\gamma_{5}+r}{2} \delta_{\xi-1, \xi} \delta_{\bar{x}, \bar{x}^{\prime}}^{(4)} \\
&+(-5 r+M(\xi)) \delta_{x, x^{\prime}}^{(5)}
\end{aligned}
$$

where we have used the notation $x \equiv(\bar{x}, \xi)$ and eq. (18) incorporates the Ansatz in eq. (12).

We see that, for large $\beta$ and $\beta^{\prime}$, the saddle point equations (13) and (14) are insensitive to the presence of fermions, leading to the solution $v=v^{\prime}=1$. There is also a strong coupling phase, with $v=v^{\prime}=0$. Most interestingly, one also finds a solution with $v \neq 0, v^{\prime}=0$; this is a layered phase, as in the pure gauge case [6], since $v^{\prime}=0$ renders the fermion propagator $G$ local in $\xi$. Hence the matrix element $j_{5}(\xi)$ vanishes, consistent with $v^{\prime}=0$ from eqs. (13) and (14)-there is a layered phase with a fermion propagator that is strictly confined to four-dimensional layers. This propagator is vectorial; and in the large $\beta$ limit (so $v=1$ ) we just have the four-dimensional Wilson propagator

$$
G^{-1}\left(p ; \xi, \xi^{\prime}\right)=\left(\mathrm{i} \sum_{\mu=1}^{4} \gamma_{\mu} \sin p_{\mu}+r \sum_{\mu=1}^{4}\left(\cos p_{\mu}-1\right)-r+M(\xi)\right) \delta_{\xi, \xi^{\prime}}
$$

\section{Summary and prospects}

Clearly the fermion propagator changes dramatically from weak coupling, i.e. $\beta, \beta^{\prime} \rightarrow \infty$, where $v=v^{\prime}=1$, to $\beta^{\prime}$ small enough (but $\beta$ large enough), where $v \neq 0$ and $v^{\prime}=0$. The localized chiral pole at weak coupling disappears entirely when $\beta^{\prime}$ is small.

Intuitively this is perfectly understandable on two counts. First: the coupling in the fifth direction becomes so strong ( $\beta^{\prime}$ small) that the chiral mode at $\xi=0$ starts to bind with its chiral partner at the boundary of the system. Second: the chiral mode at $\xi=0$ has its chirality related to the gradient of the mass profile. In the layered phase it is unable to detect the gradient and the bias for a given chirality disappears. 
What is small enough for $\beta^{\prime}$ ? In the absence of fermions, a simple argument [5] shows that $\beta_{c}^{\prime}=1 / 4$. Including fermions raises this value. For $\beta$ one finds, in the absence of fermions, $\beta_{c} \approx 1$; this holds in the presence of fermions as well. In the mass range that admits chiral fermions in the weak coupling phase, we find shifts of the order of $10 \%$ (cf. Figure 1). Note that the dotted line separating the phases $B$ and $C$ when fermions are included is only indicative of what happens close to the triple point; while it can be shown that its slope close to this point is negative, we haven't mapped it out completely.

What one must do is to unravel the surface effect, proportional to $L_{\perp}^{4}$ (where $L_{\perp}$ is the size of the system in the 4 physical directions) as proposed in ref. [10]. To this end, take the free energy of the system with a constant positive (negative) mass, i.e. $F_{+}\left(F_{-}\right)$. On the other hand, the free energy, $F_{+-}$, of the system with the mass profile is

$$
F_{+-}=L L_{\perp}^{4} f_{+-}+L_{\perp}^{4} \sigma_{+-}+\cdots
$$

Since the fermion is heavy $f_{+-}$is additive so that

$$
f_{+-}=\frac{f_{+}+f_{-}}{2} \text {. }
$$

Therefore the quantity

$$
F_{+-}-\frac{F_{+}+F_{-}}{2}
$$

gives the excess in free energy of the defect. This free energy excess should be due to a chiral theory; as such it will be the gauge field average of the effective action where the gauge invariant part is due to the $\eta$ invariant [12]. This is under investigation.

\section{Acknowledgements}

C.P.K.-A. thanks the theory division of KEK (Tsukuba, Japan) for its warm hospitality and S. Aoki, G. 't Hooft, T. Kashiwa, Y. Kikugawa, H. B. Nielsen, N. Ninomiya, M. Okawa and J. Smit for useful discussions. The work of J.P. has been supported in part by CICYT, Spain, under Grant No AEN90-0040. He is also indebted to the Spanish Ministerio de Educación y Ciencia for a fellowship.

\section{References}

[1] L. H. Karsten and J. Smit, Nucl. Phys. B144 (1978) 536

[2] H. B. Nielsen and N. Ninomiya, Nucl. Phys. B185 (1981) 20 
[3] "Non-perturbative aspects of Chiral Gauge Theories", L. Maiani, G. C. Rossi and M. Testa eds., Nucl. Phys. B (Proc. Supp.) 29C (1992)

[4] D. Kaplan, Phys. Lett. B288 (1992) 342

[5] C. G. Callan and J. A. Harvey, Nucl. Phys. B250 (1985)

[6] Y. Fu and H. B. Nielsen, Nucl. Phys. B236 (1984) 167

[7] K. Jansen and M. Schmaltz, Phys Lett. B296 (1992)

[8] M. Golterman, K. Jansen and D. Kaplan, Phys. Lett. B301 (1993) 219

[9] R. Jackiw and C. Rebbi, Phys. Rev. D13 (1976) 3398

[10] R. Narayanan and H. Neuberger, Phys. Lett. B302 (1993) 62

[11] J. M. Drouffe, Phys. Lett. B105 (1981) 46

[12] L. Alvarez-Gaumé, S. Della Pietra and V. Della Pietra, Phys. Lett. B166 (1986) 177 


\section{Figure Caption}

Figure 1: $\quad$ Phase diagram of the model. Solid (dotted) lines are for the absence (presence) of fermions. The three phases present are: a layered phase $(A)$, a weak coupling phase $(B)$ and a strong coupling phase $(C)$. 
•

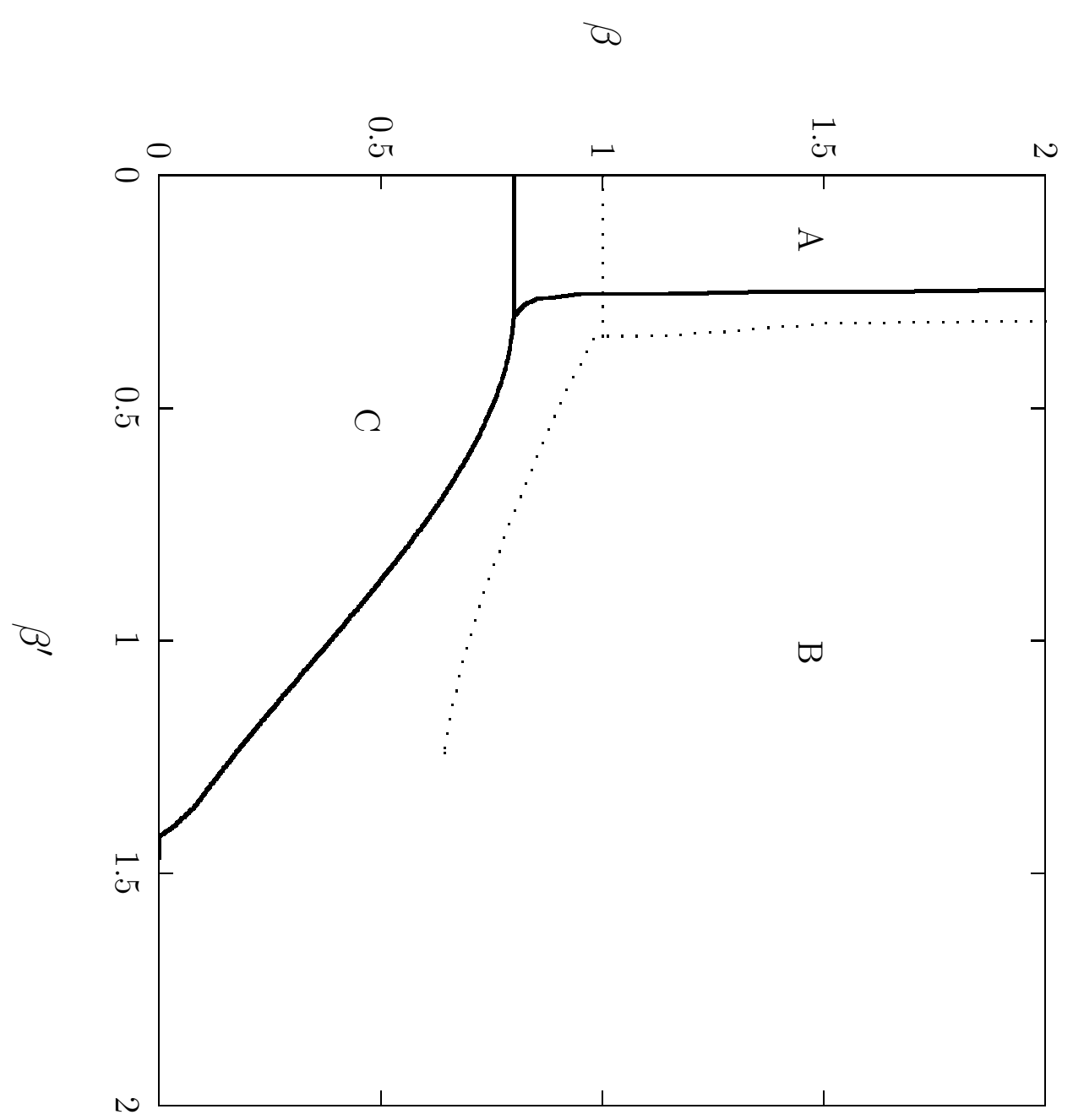

